\title{
Predictors of Small Intestinal Bacterial Overgrowth in Symptomatic Patients Referred for Breath Testing
}

\author{
Jeremy Liu Chen Kiowa, b, Ratiba Bellila ${ }^{a}$, Amelie Therrien ${ }^{a}$, \\ Sacha Sidani ${ }^{\mathrm{a}}$, Mickael Bouin ${ }^{\mathrm{a}}$
}

\begin{abstract}
Background: Indications for a breath test (BT) are well established in the symptomatic patient with risk factors predisposing them to small intestinal bacterial overgrowth (SIBO). Characteristics and the profile of this population are not well known. Our objective was to study the characteristics of patients undergoing a BT for SIBO and to identify factors associated with a positive BT.
\end{abstract}

Methods: Retrospective study was conducted from 2012 to 2016 at the neurogastroenterology unit of the Centre Hospitalier de l'Universite de Montreal (CHUM). All patients who completed a BT (lactulose and/or glucose) were included. Demographics and clinical factors were analyzed to identify predictors of positive BT. Type of antibiotic treatment and clinical response were compiled. Groups of patients with $\left(\mathrm{SIBO}^{+}\right)$ and without SIBO (SIBO-) were also compared.

Results: A total of 136 patients were included in the study (mean age 51.2 , range 20 - 80 years; $63 \%$ women), and SIBO was detected in $33.8 \%(n=46)$. Both groups were similar in terms of age, body mass index, and gender. SIBO was significantly associated with the presence of abdominal pain (odds ratio $(\mathrm{OR})=4.78 ; \mathrm{P}<0.05$ ), bloating $(\mathrm{OR}=5.39 ; \mathrm{P}<0.05)$, smoking $(\mathrm{OR}=6.66 ; \mathrm{P}<0.05)$, and anemia $(\mathrm{OR}=4.08 ; \mathrm{P}<0.05)$. No association was identified with gender, age, obesity, and risk factors for SIBO. Antibiotics were used in $43 \%$ of patients with a positive BT, but clinical response was not significantly different in the subgroup that received antibiotics versus the subgroup that did not.

Conclusions: The prevalence of SIBO is high in symptomatic patients who underwent breath testing. Abdominal pain, bloating, smoking, and anemia are strongly associated with SIBO. Treatment of SIBO with antibiotics needs to be further investigated to better determine its efficacy on gastrointestinal symptoms.

Manuscript submitted August 11, 2020, accepted September 3, 2020

Published online September 21, 2020

aDepartment of Gastroenterology, Centre Hospitalier de l'Universite de Montreal (CHUM), Montreal, QC, Canada

${ }^{\mathrm{b} C}$ Corresponding Author: Jeremy Liu Chen Kiow, Department of Gastroenterology, Department of Medicine, Centre Hospitalier de l'Universite de Montreal, 1051, Sanguinet, Montreal, QC H2X 3E4, Canada.

Email: jeremy.liu.chen.kiow@umontreal.ca
Keywords: Small intestinal bacterial overgrowth; Breath test; Gastrointestinal symptoms; Predictors; Antibiotics

\section{Introduction}

Small intestinal bacterial overgrowth (SIBO) is a condition in which colonic bacteria are seen in excess $\left(>10^{5}\right.$ colony-forming units $(\mathrm{CFU}) / \mathrm{mL})$ in the small intestine causing inflammation and malabsorption [1,2]. SIBO, sometimes asymptomatic, usually presents with symptoms of bloating, flatulence, abdominal pain, diarrhea, fatigue, and vitamin deficiencies [3]. Several conditions predispose to SIBO including intestinal stasis due to altered anatomy (abdominal surgeries, fistulas, stenoses), decreased antibacterial protection mechanisms (exocrine pancreatic insufficiency, achlorhydria) and functional intestinal stasis (dysmotility in numerous chronic diseases). SIBO is therefore associated with multiple conditions such as scleroderma, irritable bowel syndrome, inflammatory bowel diseases, chronic pancreatitis, celiac disease and cirrhosis due to multiple etiologies: alcohol, viruses, primary biliary cholangitis [3-16]. It can also be present in older individuals without any underlying condition [3].

Culture of jejunal aspirates has long been considered the gold standard in diagnosing SIBO, but it is an invasive technique and is not routinely available. Breath tests (BTs), however, are non-invasive and validated for the diagnosis of SIBO [17-19]. Indications for a BT, whether it is a lactulose breath test (LBT) or glucose breath test (GBT), are well established in the symptomatic patient with risk factors predisposing them to SIBO. However, the characteristics and profile of this population are not well known. Although antibiotics are the mainstay of therapy for SIBO, the choice, dosage, and duration of antibiotic therapy are not standardized [3].

Being a reference center for digestive motility in the province of Quebec, our main objective was to study the characteristics of patients undergoing a BT for SIBO and to identify certain clinical and paraclinical factors associated with a positive BT. The clinical response to antibiotic therapy in SIBO was also assessed.

\section{Patients and Methods}

This retrospective single center study was conducted in the 
Department of Gastroenterology of the Centre Hospitalier de l'Universite de Montreal (CHUM) in Montreal, Quebec, Canada. The study protocol conforms to the ethical guidelines of the 1975 Declaration of Helsinki and was approved by the Ethics Committee of our institution.

\section{Carbohydrate BT and SIBO diagnosis}

Assessment for the presence or absence of SIBO was carried out using a BT, LBT and/or GBT. Both are simple, affordable, non-invasive, standardized, and validated methods for measuring the orocecal intestinal transit and in turn the detection of SIBO [17-19].

A BT was done in patients with a clinical suspicion of SIBO and presenting non-specific gastrointestinal symptoms such as bloating, abdominal pain and diarrhea. All participants had their basal hydrogen and methane level in breath measured and expressed in part per million (ppm) using the Quintron $12 \mathrm{i}$ Microlyser Plus system (Milwaukee, WI, USA). Then, depending on the BT chosen by the referring physician, they ingested either $10 \mathrm{~g}$ of lactulose for the LBT or $75 \mathrm{~g}$ of glucose for the GBT and had both hydrogen and methane in breath measured every $15 \mathrm{~min}$ for a total duration of $3 \mathrm{~h}$.

Lactulose, once ingested, is not digested by the enzymes in the intestine and is fermented by colonic bacteria once it reaches the cecum, producing a hydrogen and/or methane peak that can be measured in breath [20]. The hydrogen or methane molecule passes through the blood by diffusion and then into the exhaled air, where it can be measured. The time required for a peak in hydrogen production to occur represents orocecal transit time, normally between 2 and $3 \mathrm{~h}$. In the presence of SIBO, lactulose is fermented earlier (before $90 \mathrm{~min}$ after ingestion) in the small intestine instead of the cecum, giving rise to an earlier peak in hydrogen or methane production [21]. Glucose is absorbed in the proximal part of the small intestine. Similar to the LBT, a peak in hydrogen or methane in breath is suggestive of SIBO [21].

Data were collected and the presence of SIBO was determined if a peak in hydrogen or methane production occurred earlier than $90 \mathrm{~min}$. A peak was defined as an increase in the level of hydrogen of more than $20 \mathrm{ppm}$ above the basal level and $10 \mathrm{ppm}$ for methane $[1,21]$. The interpretation of each BT and the diagnosis of SIBO were done by gastroenterologists of our institution with expertise in neurogastroenterology and digestive motility. Patients were then classified into two separate groups: one with a BT consistent with SIBO and one with BT not consistent with SIBO.

After breath testing, all patients were then referred to their physician who decided whether or not treatment was clinically indicated. Choice of antibiotic regimen, if appropriate, was left to the discretion of the treating physician.

\section{Patients}

To be included in the study, patients had to be referred to the neurogastroenterology and motility unit for a BT between June
2012 and June 2016. Patients who did not undergo breath testing after referral were excluded from the study.

\section{Data collection}

Medical charts were carefully reviewed in order to collect relevant clinical and paraclinical data. All of the following data were collected: age, gender, body mass index (BMI), presence of gastrointestinal symptoms (such as abdominal pain, diarrhea, and bloating), presence of associated comorbidities (such as celiac disease, scleroderma, pancreatitis, cirrhosis, anemia), habits (smoking and alcohol consumption), proton pump inhibitor (PPI) use, antibiotic treatment for SIBO, and clinical response to antibiotic treatment for SIBO. No physical or paraclinical exam was performed to complete the missing data. Missing data were noted as incomplete.

\section{Statistical analysis}

A descriptive statistical analysis of all the collected data was performed as well as an analysis of variance (ANOVA) to test the associations of quantitative variables such as age, weight, and BMI with positive BT by comparing the characteristics of the study sample in both positive and negative BT subgroups. Thereafter, a univariate and multivariate logistic regression analysis was completed to examine the association of the predictor variables and positive BT. Predictor variables were age, gender, BMI, smoking, gastrointestinal symptoms, comorbidities associated with SIBO, and PPI intake. Statistical calculations were done with SPSS [22]. A P value of $<0.05$ was used to define statistical significance in the final models.

\section{Results}

\section{Clinical characteristics and prevalence of SIBO}

Out of 150 patients who were referred to the neurogastroenterology and motility unit for breath testing, 136 patients were included in the study (mean age 51.2, range 20 - 80 years). The patients who finally refused to undergo breath testing or who did not comply with instructions were excluded from the study. The prevalence of SIBO in the patients who underwent breath testing was $33.8 \%$, 46 BT out of 136 that were compatible with SIBO. Fourteen patients were excluded because they did not undergo breath testing after being referred. Baseline characteristics are presented in Table 1. The SIBO group was similar to the group without SIBO in terms of age (mean age 52.7, range 20 - 80 vs. 50.49, range 23 - 78 years; $\mathrm{P}=$ non-significant (NS)), BMI (24.88, range $17.58-33.33$ vs. 25.41 , range $14.53-52.47$ $\left.\mathrm{kg} / \mathrm{m}^{2} ; \mathrm{P}=\mathrm{NS}\right)$ and gender $(63 \%$ women in both cases, $\mathrm{P}=\mathrm{NS})$.

\section{Associations between SIBO (positive BT) and clinical factors}

From the clinical characteristics of patients, a significant asso- 
Table 1. Characteristics of Patients Who Completed a BT: Comparison Between the Two Groups SIBO- and SIBO+

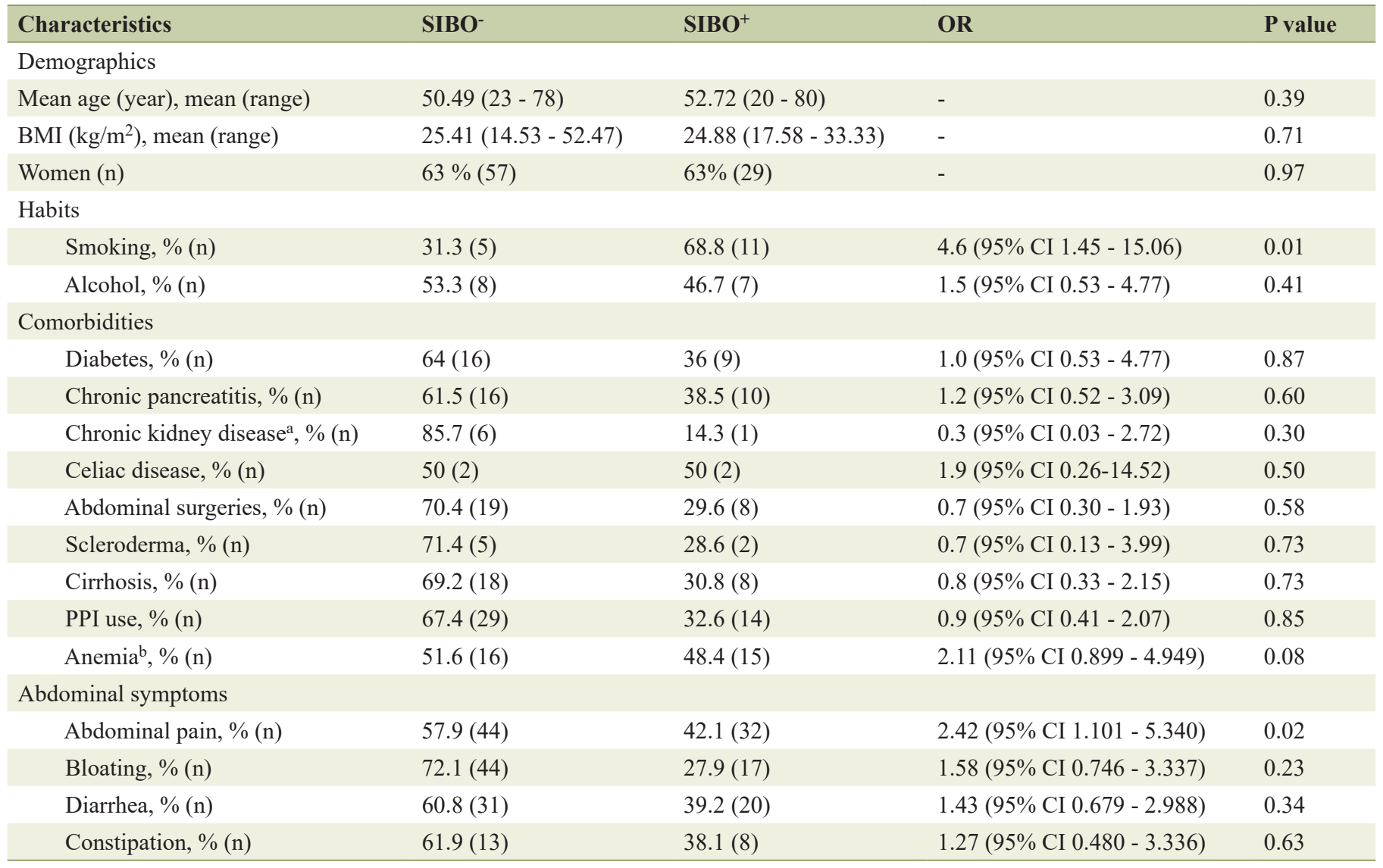

BT: breath test; SIBO: small intestinal bacterial overgrowth; SIBO-: group without SIBO; $\mathrm{SIBO}^{+}$: group with SIBO; BMI: body mass index; PPIs: proton pump inhibitors; OR: odds ratio; $\mathrm{Cl}$ : confidence interval. ${ }^{a}$ Chronic kidney disease (kidney damage or glomerular filtration rate $<60 \mathrm{~mL} / \mathrm{min} / 1.73 \mathrm{~m}{ }^{2}$ for at least 3 months); ${ }^{b} H e m o g l o b i n ~<140 \mathrm{~g} / \mathrm{L}$ in men and $<120 \mathrm{~g} / \mathrm{L}$ in women.

ciation was observed by univariate logistic regression between positive $\mathrm{BT}$ and smoking $(\mathrm{OR}=4.6 ; \mathrm{P}<0.05)$ and abdominal pain $(\mathrm{OR}=2.42 ; \mathrm{P}<0.05)$ (Table 1$)$. Interestingly, multivariate analysis showed that this association remained strongly positive and that two additional factors associated with a positive $\mathrm{BT}$ have been identified, anemia $(\mathrm{OR}=4.08 ; \mathrm{P}<0.05)$ and bloating $(\mathrm{OR}=5.39 ; \mathrm{P}<0.05)$ (Table 2). No association was identified with gender, age, obesity, and risk factors for SIBO.

\section{Type of antibiotic treatment for SIBO and its clinical re- sponse}

Antibiotics were used in $43 \%$ of patients with a positive BT and $2.2 \%$ of patients with a negative BT (Fig. 1). The most commonly prescribed antibiotic in patients was metronidazole (Table 3). However, no difference was observed in symptom improvement in patients with or without antibiotic treatment.

\section{Discussion}

In this study, 46 out of 136 BT (LBT and/or BT) were compat- ible with SIBO (33.8\%). A positive BT was strongly associated with the presence of abdominal pain, bloating, smoking, and anemia. Interestingly, there was no association with the chronic diseases known to be associated with SIBO (such as cirrhosis, scleroderma, celiac disease, chronic pancreatitis). Furthermore, among the 20 patients with a positive BT, only nine (45\%) saw clinical improvement in gastrointestinal symptoms.

Our results demonstrate that the high occurrence of SIBO remains comparable to recently published studies on the prevalence of SIBO in patients with chronic pancreatitis [23-25], scleroderma [6], celiac disease $[15,26]$, and primary biliary cholangitis [16]. Although our study did not show a statistically significant association between SIBO and these pathologies, it should be sought in these patients because of its high prevalence.

Culture of jejunal aspirates has long been considered the gold standard in diagnosing SIBO, but it is not routinely available and is invasive. BTs, on the other hand, are more readily available, non-invasive and validated for the diagnosis of SIBO $[21,27]$. Both the LBT and GBT have similar specificity and sensitivity, with the LBT being slightly more specific and GBT more sensitive $[20,27]$. In order to increase both the sensitivity and specificity for the diagnosis of SIBO, we in- 
Table 2. Association Between Characteristics and $\mathrm{SIBO}^{+}$After Multivariate Analysis

\begin{tabular}{lll} 
Characteristics & OR & P value \\
\hline Bloating & 5.39 & 0.01 \\
Abdominal pain & 4.78 & 0.01 \\
Anemia & 4.08 & 0.02 \\
Smoking & 6.66 & 0.01 \\
\hline
\end{tabular}

Factors adjusted for in the multivariate analysis: age, gender, bloating, abdominal pain, constipation, diarrhea, weight loss, anemia, and smoking. SIBO: small intestinal bacterial overgrowth; SIBO+: group with SIBO; OR: odds ratio.

cluded patients who had an LBT and/or GBT when available. Furthermore, both hydrogen and methane were measured systematically in the BTs we analyzed. This is important because it is well recognized that some individuals produce methane instead of hydrogen in the presence of SIBO [20,27]. This was done in an attempt to reduce the amount of possible false negatives. However, in our study, all participants with a positive BT produced peaks in hydrogen instead of methane.

Patients with a positive BT in our study were predominantly female (63\%). However, no demographic characteristics were predictive of SIBO. Patients over 65 years old were no more likely to have positive BT. Although studies in the elderly suggest an association between age and SIBO [28, 29], this association is not always identified [30]. Many factors such as intrinsic changes in intestinal motility, hypochlorhydria, the presence of intestinal diverticula, polypharmacy (with medications affecting gastrointestinal motility), and the higher prevalence of comorbidities in patients over 65 years old put them at higher risk of SIBO [3, 17]. Nevertheless, these same factors can be found also in the non-elderly population.

There is a controversy in the literature about the association between obesity and SIBO. Our study did not find an association between obesity and a positive BT. Schatz et al demonstrated an inverse association between BMI and SIBO [31]. One possible explanation for this association could be that SIBO causes malabsorption and weight loss. However, many studies suggest that alteration in enteric flora may have an impact on BMI. An association between SIBO and morbid

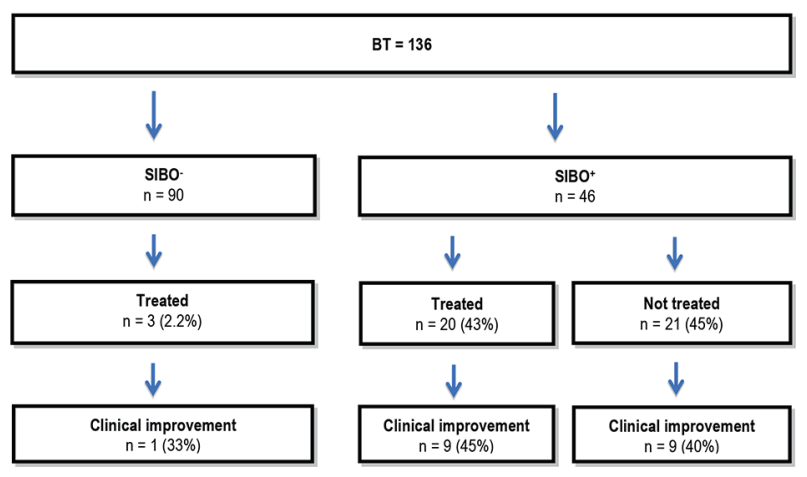

Figure 1. Antibiotic treatment. BT: breath test; SIBO: small intestinal bacterial overgrowth; SIBO: group without SIBO; SIBO+: group with SIBO.
Table 3. Antibiotics Chosen for SIBO Treatment

\begin{tabular}{lll}
\hline Antibiotics & $\mathbf{n = 3 8}$ & $\mathbf{\%}$ \\
\hline Metronidazole & 15 & $39.5 \%$ \\
Ciprofloxacin & 4 & $10.5 \%$ \\
Amoxicillin and clavulanate & 4 & $10.5 \%$ \\
Amoxicillin & 3 & $7.9 \%$ \\
Doxycycline & 5 & $13.2 \%$ \\
Rifaximin & 3 & $7.9 \%$ \\
Tetracycline & 1 & $2.6 \%$ \\
Minocycline & 1 & $2.6 \%$ \\
Norfloxacin & 1 & $2.6 \%$ \\
Trimethoprim-sulfamethoxazole & 1 & $2.6 \%$ \\
\hline
\end{tabular}

SIBO: small intestinal bacterial overgrowth.

obesity in a population referred for bariatric surgery has been reported [3]. This variability suggests that the relationship between SIBO and obesity is much more complex. It is important to mention that only seven subjects in our study sample had a BMI over 30, which could have led to an underestimation of SIBO at higher BMI levels.

Bloating and abdominal pain were good predictors of a positive BT and therefore SIBO. This is in accordance with the fact that they are cardinal symptoms of SIBO. Anemia is recognized as a consequence of SIBO and is explained mainly by vitamin B12 deficiency [32]. Moreover, in addition to having objectified a positive association of anemia with SIBO, three out of four patients with vitamin B12 deficiency had a positive BT.

The association of SIBO with the use of PPI has recently been of significant clinical interest since their use is very common and some gastrointestinal symptoms similar to SIBO such as diarrhea, boating, and the feeling of fullness have been reported in patients who are chronic PPI users. However, this association still remains controversial [23, 33, 34]. Indeed, several factors independent of SIBO could cause a high prevalence of these gastrointestinal symptoms in PPI users.

Our study demonstrated a strongly positive association between being an active smoker and the occurrence of SIBO. To our knowledge, no study has been conducted to investigate the relationship between active smoking and SIBO. Opstelten et al demonstrated a reduction in intestinal microbial diversity in smokers compared to non-smokers with Crohn's disease, which may predispose them to have an exacerbation of their condition [35]. Our study confirms that smoking is probably another risk factor for SIBO; the mechanism could be an alteration of the intestinal microbiome by tobacco or the fact that smokers have comorbidities that could influence the positivity of BT. However, it is possible that the positivity in smokers is due to non-compliance with the instructions to refrain from smoking before the test.

Clinical improvement after antibiotic treatment was similar in patients treated for SIBO compared to untreated patients. There was some heterogeneity of the antibiotics used, but metronidazole, ciprofloxacin, and amoxicillin and clavulanate were the most commonly chosen antibiotics to treat for SIBO. 
Some patients had multiple courses of antibiotics for treatment of SIBO. In our sample, the clinical improvement in treated patients may be underestimated by the small number of patients included in both the treated and untreated subgroups with a positive BT. The small sample, as well as the retrospective nature of the study, unfortunately limits the power of our study to accurately determine clinical response to antibiotic treatment. Furthermore, the major disadvantage of a BT is that it does not identify the bacteria that cause the symptoms of SIBO, which makes the choice and duration of antibiotic treatment difficult [2]. Therefore, it is possible that patients did not have an adequate treatment adapted to their specific conditions. Only a few patients had rifaximin as a treatment for SIBO. Another point to consider is that the improvement was only judged by clinical parameters (abdominal pain, bloating, and diarrhea). A proportion of patients who reported not having any improvement could possibly have a negative BT after treatment. In that case, the lack of clinical response could simply be symptoms unrelated to SIBO. However, GBT positivity recurrence rate seems to be high despite appropriate antibiotic treatment and is associated with gastrointestinal symptoms relapse compatible with SIBO recurrence [36]. Finally, it is important to note that after their BT, all patients were referred to their physician who then decided whether or not treatment was clinically indicated. In fact, since the choice of antibiotic regimen was left to the discretion of the treating physician, there was no control over the treatment or not of our patients. Indeed, this could also explain our negative results concerning antibiotic treatment on SIBO symptoms.

This study demonstrates the experience of a tertiary center in neurogastroenterology and motility with a population at high risk for SIBO. However, it does include certain limitations including its retrospective nature, a study population specifically selected and referred to a single tertiary care center, the subjective assessment of clinical response after SIBO treatment, and the lack of randomization of treatment options (antibiotics versus no antibiotics).

\section{Conclusions}

In summary, we can conclude from our study that there is a high prevalence of SIBO in symptomatic patients who undergo breath testing. Abdominal pain, bloating, smoking, and anemia are strongly associated with the occurrence of SIBO in symptomatic patients referred for breath testing. Antibiotic treatment of SIBO needs to be further investigated to determine its efficacy on gastrointestinal symptoms and to better characterize the type of patients who would benefit from it.

\section{Acknowledgments}

None to declare.

\section{Financial Disclosure}

None to declare.

\section{Conflict of Interest}

None to declare.

\section{Informed Consent}

Not applicable.

\section{Author Contributions}

JLCK, RB, AT, SS and MB contributed to study concept and design; RB contributed to acquisition of data, analysis, interpretation of data and statistical analysis; JLCK drafted the manuscript; JLCK, RB, AT and MB were involved in critical revision of the manuscript for important intellectual content; MB was responsible for study supervision.

\section{Data Availability}

Any inquiries regarding supporting data availability of this study should be directed to the corresponding author.

\section{Abbreviations}

SIBO: small intestinal bacterial overgrowth; BT: breath test; LBT: lactulose breath test; GBT: glucose breath test; ppm: parts per million; $\mathrm{SIBO}^{+}$: groups of patients with $\mathrm{SIBO}$; $\mathrm{SIBO}^{-}$ : groups of patients without SIBO; OR: odds ratio; CFU: colony-forming units; ppm: part per million; BMI: body mass index; PPI: proton pump inhibitor; ANOVA: analysis of variance; NS: non-significant

\section{References}

1. Khoshini R, Dai SC, Lezcano S, Pimentel M. A systematic review of diagnostic tests for small intestinal bacterial overgrowth. Dig Dis Sci. 2008;53(6):1443-1454.

2. Gasbarrini A, Lauritano EC, Gabrielli M, Scarpellini E, Lupascu A, Ojetti V, Gasbarrini G. Small intestinal bacterial overgrowth: diagnosis and treatment. Dig Dis. 2007;25(3):237-240.

3. Bures J, Cyrany J, Kohoutova D, Forstl M, Rejchrt S, Kvetina J, Vorisek V, et al. Small intestinal bacterial overgrowth syndrome. World J Gastroenterol. 2010;16(24):2978-2990.

4. Trespi E, Ferrieri A. Intestinal bacterial overgrowth during chronic pancreatitis. Curr Med Res Opin. 1999;15(1):4752.

5. Lewis SJ, Franco S, Young G, O'Keefe SJ. Altered bowel function and duodenal bacterial overgrowth in patients treated with omeprazole. Aliment Pharmacol Ther. 1996;10(4):557-561.

6. Marie I, Ducrotte P, Denis P, Menard JF, Levesque H. 
Small intestinal bacterial overgrowth in systemic sclerosis. Rheumatology (Oxford). 2009;48(10):1314-1319.

7. Reddymasu SC, McCallum RW. Small intestinal bacterial overgrowth in gastroparesis: are there any predictors? J Clin Gastroenterol. 2010;44(1):e8-13.

8. Wedlake L, Thomas K, McGough C, Andreyev HJ. Small bowel bacterial overgrowth and lactose intolerance during radical pelvic radiotherapy: An observational study. Eur J Cancer. 2008;44(15):2212-2217.

9. Esposito I, de Leone A, Di Gregorio G, Giaquinto S, de Magistris L, Ferrieri A, Riegler G. Breath test for differential diagnosis between small intestinal bacterial overgrowth and irritable bowel disease: an observation on non-absorbable antibiotics. World J Gastroenterol. 2007;13(45):6016-6021.

10. Posserud I, Stotzer PO, Bjornsson ES, Abrahamsson H, Simren M. Small intestinal bacterial overgrowth in patients with irritable bowel syndrome. Gut. 2007;56(6):802-808.

11. Klaus J, Spaniol U, Adler G, Mason RA, Reinshagen M, von Tirpitz CC. Small intestinal bacterial overgrowth mimicking acute flare as a pitfall in patients with Crohn's Disease. BMC Gastroenterol. 2009;9:61.

12. Bauer TM, Steinbruckner B, Brinkmann FE, Ditzen AK, Schwacha H, Aponte JJ, Pelz K, et al. Small intestinal bacterial overgrowth in patients with cirrhosis: prevalence and relation with spontaneous bacterial peritonitis. Am J Gastroenterol. 2001;96(10):2962-2967.

13. Sabate JM, Jouet P, Harnois F, Mechler C, Msika S, Grossin M, Coffin B. High prevalence of small intestinal bacterial overgrowth in patients with morbid obesity: a contributor to severe hepatic steatosis. Obes Surg. 2008;18(4):371-377.

14. Pimentel M, Wallace D, Hallegua D, Chow E, Kong Y, Park S, Lin HC. A link between irritable bowel syndrome and fibromyalgia may be related to findings on lactulose breath testing. Ann Rheum Dis. 2004;63(4):450-452.

15. Rubio-Tapia A, Barton SH, Rosenblatt JE, Murray JA. Prevalence of small intestine bacterial overgrowth diagnosed by quantitative culture of intestinal aspirate in celiac disease. J Clin Gastroenterol. 2009;43(2):157161.

16. Liu Chen Kiow J, Vincent C, Sidani S, Bouin M. High occurrence of small intestinal bacterial overgrowth in primary biliary cholangitis. Neurogastroenterol Motil. 2019;31(11):e13691.

17. Gabrielli M, D'Angelo G, Di Rienzo T, Scarpellini E, Ojetti V. Diagnosis of small intestinal bacterial overgrowth in the clinical practice. Eur Rev Med Pharmacol Sci. 2013; 17(Suppl 2):30-35.

18. Urita $\mathrm{Y}$, Ishihara $\mathrm{S}$, Akimoto $\mathrm{T}$, Kato $\mathrm{H}$, Hara N, Honda Y, Nagai Y, et al. Seventy-five gram glucose tolerance test to assess carbohydrate malabsorption and small bowel bacterial overgrowth. World J Gastroenterol. 2006;12(19):3092-3095.

19. Kerlin P, Wong L. Breath hydrogen testing in bacterial overgrowth of the small intestine. Gastroenterology. 1988;95(4):982-988.

20. Leung Ki EL, Roduit J, Delarive J, Guyot J, Michetti P, Dorta G. Small intestine bacterial overgrowth. Rev Med
Suisse. 2010;6(233):186-188, 190-181.

21. Rezaie A, Buresi M, Lembo A, Lin H, McCallum R, Rao S, Schmulson M, et al. Hydrogen and methanebased breath testing in gastrointestinal disorders: the North American consensus. Am J Gastroenterol. 2017;112(5):775-784.

22. IBM Corp. Released 2016. IBM SPSS Statistics for Windows, Version 24.0. Armonk, NY: IBM Corp.

23. Choung RS, Ruff KC, Malhotra A, Herrick L, Locke GR, 3rd, Harmsen WS, Zinsmeister AR, et al. Clinical predictors of small intestinal bacterial overgrowth by duodenal aspirate culture. Aliment Pharmacol Ther. 2011;33(9):1059-1067.

24. Capurso G, Signoretti M, Archibugi L, Stigliano S, Delle Fave G. Systematic review and meta-analysis: Small intestinal bacterial overgrowth in chronic pancreatitis. United European Gastroenterol J. 2016;4(5):697-705.

25. Therrien A, Bouchard S, Sidani S, Bouin M. Prevalence of small intestinal bacterial overgrowth among chronic pancreatitis patients: a case-control study. Can J Gastroenterol Hepatol. 2016;2016:7424831.

26. Losurdo G, Marra A, Shahini E, Girardi B, Giorgio F, Amoruso A, Pisani A, et al. Small intestinal bacterial overgrowth and celiac disease: A systematic review with pooled-data analysis. Neurogastroenterol Motil. 2017;29(6):e13028.

27. Gasbarrini A, Corazza GR, Gasbarrini G, Montalto M, Di Stefano M, Basilisco G, Parodi A, et al. Methodology and indications of $\mathrm{H} 2$-breath testing in gastrointestinal diseases: the Rome Consensus Conference. Aliment Pharmacol Ther. 2009;29(Suppl 1):1-49.

28. Elphick DA, Chew TS, Higham SE, Bird N, Ahmad A, Sanders DS. Small bowel bacterial overgrowth in symptomatic older people: can it be diagnosed earlier? Gerontology. 2005;51(6):396-401.

29. Parlesak A, Klein B, Schecher K, Bode JC, Bode C. Prevalence of small bowel bacterial overgrowth and its association with nutrition intake in nonhospitalized older adults. J Am Geriatr Soc. 2003;51(6):768-773.

30. Newberry C, Tierney A, Pickett-Blakely O. Lactulose hydrogen breath test result is associated with age and gender. Biomed Res Int. 2016;2016:1064029.

31. Schatz RA, Zhang Q, Lodhia N, Shuster J, Toskes PP, Moshiree B. Predisposing factors for positive D-Xylose breath test for evaluation of small intestinal bacterial overgrowth: a retrospective study of 932 patients. World J Gastroenterol. 2015;21(15):4574-4582.

32. Giannella RA, Broitman SA, Zamcheck N. Vitamin B12 uptake by intestinal microorganisms: mechanism and relevance to syndromes of intestinal bacterial overgrowth. J Clin Invest. 1971;50(5):1100-1107.

33. Lombardo L, Foti M, Ruggia O, Chiecchio A. Increased incidence of small intestinal bacterial overgrowth during proton pump inhibitor therapy. Clin Gastroenterol Hepatol. 2010;8(6):504-508.

34. Lo WK, Chan WW. Proton pump inhibitor use and the risk of small intestinal bacterial overgrowth: a metaanalysis. Clin Gastroenterol Hepatol. 2013;11(5):483490. 
35. Opstelten JL, Plassais J, van Mil SW, Achouri E, Pichaud M, Siersema PD, Oldenburg B, et al. Gut microbial diversity is reduced in smokers with Crohn's disease. Inflamm Bowel Dis. 2016;22(9):2070-2077.
36. Lauritano EC, Gabrielli M, Scarpellini E, Lupascu A, Novi M, Sottili S, Vitale G, et al. Small intestinal bacterial overgrowth recurrence after antibiotic therapy. Am J Gastroenterol. 2008;103(8):2031-2035. 